\title{
ON THE STRONG LAW OF LARGE NUMBERS
}

\author{
BY \\ P. ERDÖS
}

In the present note $f(x),-\infty<x<\infty$, will denote a function satisfying the following conditions: (1) $f(x+1)=f(x)$, (2) $\int_{0}^{1} f(x)=0, \int_{0}^{1} f(x)^{2}=1$. By $n_{1}<n_{2}$ $<\cdots$ we shall denote an arbitrary sequence satisfying $n_{k+1} / n_{k}>c>1$, and by $S_{n}(f)$ the $n$th partial sum of the Fourier series of $f(x)$.

In a recent paper $\mathrm{Kac}$, Salem, and $Z_{y g m u n d}\left(^{1}\right)$ prove (among others) that if for some $\epsilon>0$

$$
\int_{0}^{1}\left(f(x)-\phi_{n}(f)\right)^{2}=O\left(\frac{1}{(\log n)^{\epsilon}}\right),
$$

then for almost all $x$

$$
\lim _{N \rightarrow \infty} \frac{1}{N}\left(\sum_{k=1}^{N} f\left(n_{k} x\right)\right)=0,
$$

or roughly speaking the strong law of large numbers holds for $f\left(n_{k} x\right.$ ) (in fact the authors prove that $\sum f\left(n_{k} x\right) / k$ converges almost everywhere).

The question was raised whether (2) holds for any $f(x)$. This was known for the case $n_{k}=2^{k}\left({ }^{2}\right)$. In the present paper it is shown that this is not the case. In fact we prove the following theorem.

THEOREM 1. There exists an $f(x)$ and a sequence $n_{k}$ so that for almost all $x$

$$
\limsup _{N \rightarrow \infty} \frac{1}{N}\left(\sum_{k=1}^{N} f\left(n_{k} x\right)\right)=\infty .
$$

Further we prove the following sharpening of the result of Kac-SalemZygmund:

TheOREM 2. Assume that for some $\epsilon>0$

$$
\int_{0}^{1}\left(f(x)-\phi_{n}(f)\right)^{2}=O\left(\frac{1}{(\log \log n)^{2+\epsilon}}\right)
$$

then (2) holds.

By a slight modification of the construction of the $f(x)$ of Theorem 1 it is easy to construct an $f(x)$ and a sequence $n_{k}$ for which (3) holds and for which

Presented to the Society, February 28, 1948; received by the editors January 28, 1948.

(1) Trans. Amer. Math. Soc. vol. 63 (1948) pp. 235-243. 223.

(2) This result is due to Raikov. See F. Riesz, Comment. Math. Helv. vol. (17) (1944) p. 


$$
\int_{0}^{1}\left(f(x)-\phi_{n}(f)\right)^{2}<\frac{1}{(\log \log \log n)^{c}} .
$$

There is clearly a gap between (4) and (5). It seems probable that, in Theorem 2 , (4) can be replaced by $1 /(\log \log \log n)^{c_{1}}$, but much sharper methods would be needed than used here.

The following problem also seems of some interest: By an easy modification in the construction of the $f(x)$ of Theorem 1 we can show the existence of an $f(x)$ and a sequence $n_{k}$, so that for almost all $x$

$$
\limsup _{N \rightarrow \infty} \frac{1}{N(\log \log N)^{1 / 2-\epsilon}}\left(\sum_{k=1}^{N} f\left(n_{k} x\right)\right)=\infty .
$$

On the other hand we can show that for almost all $x$

$$
\lim _{N \rightarrow \infty} \frac{1}{N(\log N)^{1 / 2+\epsilon}}\left(\sum_{k=1}^{N} f\left(n_{k} x\right)\right)=0 .
$$

Again there is a gap between (6) and (7). (6) seems to give the right order of magnitude, but I can not prove this.

One final remark. The $f(x)$ of Theorem 1 is unbounded. The possibility that (2) holds for all bounded functions $f(x)$ remains open.

Proof of Theorem 1. Let $u_{k}, v_{k}$, and $A_{k}$ tend to infinity sufficiently fast (their growth will be specified later). $r_{m}(x)$ denotes the $m$ th Rademacher function $\left({ }^{3}\right)$. Put

$$
f(x)=\sum_{k=1}^{\infty} \sum_{m=u_{k}+1}^{v_{k} m} \frac{v_{m}(x)}{\left(A_{k}\left(v_{k}-u_{k}\right)\right)^{1 / 2}}, \quad \sum_{k=1}^{\infty} \frac{1}{A_{k}}=1 .
$$

Clearly the series for $f(x)$ converges almost everywhere and $\int_{0}^{1} f(x)=0$, $\int_{0}^{1} f(x)^{2}=1$. Now we define the $n_{k}$. Put $j_{k}=\left[e^{A_{k}^{3}}\right]$. Denote by $I_{t}^{(k)}$ the interval

$$
\left((2 t-1) v_{k},(2 t-1) v_{k}+l_{t}^{(k)}\right), \quad t=1,2, \cdots, j_{k},
$$

where $l_{t}^{(k)}=2 l_{l-1}^{(k)}$ and $l_{1}^{(k)}$ is very large compared to $v_{k-1}, A_{k-1}, l_{j_{k-1}}^{(k-1)}$, and will be specified later. If we choose $v_{k}>l_{j_{k}}^{(k)}$ then the $I_{t}^{(k)}$ don't overlap. The $n_{k}$ are the integers of the form $2^{m}$ where $m \subset I_{t}^{(k)}, k=1,2, \cdots ; t=1,2, \cdots, j_{k}$.

Order the $l$ 's according to their size. Clearly each $l$ is greater than the sum of all previous $l$ 's. Thus a simple argument shows that to prove (3) it will be sufficient to show that for every fixed $c$ and almost all $x$

$$
\lim \sup \frac{1}{l_{t}^{(\boldsymbol{k})}}\left(\sum_{m \subset I_{t}^{(k)}} f\left(2^{m} x\right)\right)>c, \quad k=1,2, \cdots ; t=1,2, \cdots, j_{k} .
$$

(3) Instead of $r_{m}(x)$ I originally used $\cos 2^{m} x$. The advantage of using Rademacher functions was pointed out to me by Kac. 
(Since if $m_{r+1}>2 m_{r}$, and for every $c$ lim sup $\left(1 /\left(m_{r+1}-m_{r}\right)\right) \sum_{m_{r}}^{m_{r+1}} a_{u}>c$, then $\lim \sup (1 / u) \sum_{k=1}^{u} a_{k}=\infty$. Let now $m_{r}$ be the sum of the $r$ first $l$ 's, then clearly (3) is a consequence of (9).)

Hence it will suffice to show that for every $\epsilon$ and sufficiently large $k$ the measure of the set in $x$ satisfying at least one of the inequalities

$$
\frac{1}{l_{t}^{(k)}}\left(\sum_{m \subset I_{t}^{(k)}} f\left(2^{m} x\right)\right)>c, \quad t=1,2, \cdots, j_{k},
$$

is greater than $1-\epsilon$.

Put

$$
f(x)=f_{1}(x)+f_{2}(x)+f_{3}(x)
$$

where

$$
\begin{gathered}
f_{1}(x)=\sum_{s=1}^{k-1} \sum_{m=u_{s}+1}^{v_{s}} \frac{r_{m}(x)}{\left(A_{s}\left(v_{s}-u_{s}\right)\right)^{1 / 2}}, \quad f_{2}(x)=\sum_{m=u_{k}+1}^{v_{k}} \frac{r_{m}(x)}{\left(A_{k}\left(u_{k}-v_{k}\right)\right)^{1 / 2}}, \\
f_{3}(x)=\sum_{s>k} \sum_{m=u_{s}+1}^{v_{s}} \frac{r_{m}(x)}{\left(A_{s}\left(v_{s}-u_{s}\right)\right)^{1 / 2}} .
\end{gathered}
$$

A simple calculation shows that

$$
\begin{aligned}
\sum_{m \subset I_{t}^{(k)}} f_{2}\left(2^{m} x\right) & =\frac{l_{t}^{(k)}}{\left(A_{k}\left(v_{k}-u_{k}\right)\right)^{1 / 2}} \sum r_{m}(x)+\sum_{1}+\sum_{2} \\
& =\sum+\sum_{1}+\sum_{2}
\end{aligned}
$$

where $m$ runs in the interval

$$
\left(u_{k}+(2 t-1) v_{k}+l_{t}^{(k)}, 2 t v_{k}\right)
$$

and

$$
\begin{gathered}
\sum_{1}=\sum_{a=1}^{l_{t}^{(k)}} \frac{l_{t}^{(k)}-a}{\left(A_{k}\left(v_{k}-u_{k}\right)\right)^{1 / 2}} r_{y-a}(x), \quad y=u_{k}+(2 t-1) v_{k}+l_{t}^{(k)}, \\
\sum_{2}=\sum_{a=1}^{t_{t}^{(k)}} \frac{l_{t}^{(k)}-a}{\left(A_{k}\left(v_{k}-u_{k}\right)\right)^{1 / 2}} r_{2 t v_{k}+a}(x) .
\end{gathered}
$$

Now $\sum r_{m}(x)$ is the sum of

$$
v_{k}-u_{k}-l_{t}^{(k)}>v_{k} / 2
$$

Rademacher functions (we choose $v_{k}>2\left(u_{k}+l_{l}^{(k)}\right)$ ). It is well known $\left(^{4}\right)$ that

(4) See, for example, P. Erdös, Ann. of Math. vol. 43 (1942) p. 420, formula (0.7). Incidentally the formula in question should read $c_{1}\left(x^{2} / n\right) e^{-2 x^{2} / n}<\operatorname{Pr}\left(A_{n}(x)\right)<c_{2}\left(x^{2} / n\right) e^{-2 x^{2} / n}$. 
the measure of the set in $x$ for which

$$
\sum r_{m}(x)>4 c\left(A_{k}\right)^{1 / 2}\left(v_{k}\right)^{1 / 2}
$$

is greater than

$$
c_{1} A_{k} e^{-32 c^{2} A_{k}}>e^{-A_{k}^{2}}
$$

for sufficiently large $A_{k}$. Thus the measure of the set in $x$ for which

$$
\sum=\frac{l_{t}^{(k)}}{\left(A_{k}\left(v_{k}-u_{k}\right)\right)^{1 / 2}} \sum r_{m}(x)>4 c l_{t}^{(k)}
$$

is greater than $e^{-A_{k}^{2}}$. Clearly for all $x$

$$
\left|\sum_{1}+\sum_{2}\right|<\frac{2\left(l_{t}^{(k)}\right)^{2}}{\left(A_{k}\left(v_{k}-u_{k}\right)\right)^{1 / 2}}<\frac{4\left(l_{t}^{(k)}\right)^{2}}{\left(v_{k}\right)^{1 / 2}}<1
$$

if we choose $v_{k}>16\left(l_{t}^{(k)}\right)^{4}$. Thus finally from (11), (12), and (13) the measure of the set in $x$ for which

$$
\sum_{m \subset I_{t}^{(k)}} f_{2}\left(2^{m} x\right)>4 c l_{t}^{(k)}-1>3 c l_{t}^{(k)}
$$

is greater than $e^{-A_{k}^{2}}$.

If $v_{k}>2 l_{t}^{(k)}$ for all $t$, then the functions

$$
\sum_{m \subset I_{t}^{(k)}} f_{2}\left(2^{m} x\right), \quad t=1,2, \cdots j_{k},
$$

are independent (since the same $r_{m}(x)$ does not appear in two different sums). Thus the measure of the set in $x$ for which one of the $j_{k}$ inequalities

$$
\sum_{m \subset I_{t}^{(k)}} f_{2}\left(2^{m} x\right)>3 c l_{t}^{(k)}, \quad t=1,2, \cdots, j_{k},
$$

holds, is greater than

$$
1-(1-1 / y)^{z}>1-\epsilon / 2\left(y=e^{A_{k}^{2}}, z=e^{A_{k}^{3}}\right) .
$$

Further if $l_{t}^{(k)}>v_{k-1}$

$$
\int_{0}^{1}\left(\sum_{m \subset I_{t}^{(k)}} f_{1}\left(2^{m} x\right)\right)^{2}<v_{k-1}^{2}\left(l_{t}^{(k)}+v_{k-1}\right)<2 v_{k-1}^{2} l_{t}^{(k)}
$$

since only the $r_{m}$ 's with $m \leqq l_{t}^{(k)}+v_{k-1}$ occur and the coefficients of all of them are not greater than $v_{k-1}$. Thus from Tchebychef's inequality we obtain that the measure of the set in $x$ for which one of the $j_{k}$ inequalities

$$
\sum_{m \subset I_{t}^{(k)}} f_{1}\left(2^{m} x\right)>c l_{t}^{(k)}, \quad t=1,2, \cdots, j_{k},
$$


holds is less than

$$
\sum_{t=1}^{i_{\kappa}} \frac{2 v_{k-1}^{2}}{c^{2} l_{l}^{(k)}}<\frac{4 v_{k-1}^{2}}{c l_{1}^{(k)}}<\frac{\epsilon}{4}, l_{1}^{(k)}>16 v_{k-1}^{2} / c \epsilon .
$$

Finally we have by a simple computation

$$
\int_{0}^{1}\left(\sum_{m \subset I_{t}^{(k)}} f_{3}\left(2^{m} x\right)\right)^{2}<4\left(l_{t}^{(k)}\right)^{2} \sum_{r>k} \frac{1}{A_{r}}<1
$$

if $A_{k+1}, \cdots$ are sufficiently large. Thus the measure of the set in $x$ for which one of the inequalities

$$
\sum_{m \subset I_{t}^{(k)}} f_{3}\left(2^{m} x\right)>c l_{t}^{(k)}, \quad t=1,2, \cdots, j_{k}
$$

holds is less than

$$
\sum_{t=1}^{i_{k}} \frac{1}{\left(c l_{t}^{(k)}\right)^{2}}<\frac{\epsilon}{4} .
$$

Thus finally from (15), (16), (17), (18), (19), and (20) we obtain (10) and this completes the proof of Theorem 1 .

Sketch of the Proof of Theorem 2. Put $j-i=r$, then $n_{j} / n_{i}>c^{r}$. Denote by $a_{1}, b_{1}, a_{2}, b_{2}, \cdots$ the Fourier coefficients of $f(x)$. By (4) we evidently have

$$
\begin{aligned}
\int_{0}^{1} f\left(n_{i} x\right) f\left(n_{j} x\right)=\sum_{n_{i} u=n_{j} v}\left(a_{u} a_{v}+b_{u} b_{v}\right) \leqq & \left(\sum_{k=1}^{\infty} a_{k}^{2} \sum_{k>c^{r}} a_{k}^{2}\right)^{1 / 2} \\
& +\left(\sum_{k=1}^{\infty} b_{k}^{2} \sum_{k>c^{r}} b_{k}^{2}\right)^{1 / 2}<\frac{c_{1}}{(\log r)^{1+\epsilon / 2}} .
\end{aligned}
$$

Hence

$$
\int_{0}^{1}\left(\sum_{z}^{z+N} f\left(n_{k} x\right)\right)^{2}=O\left(\frac{N^{2}}{(\log N)^{1+\epsilon / 2}}\right),
$$

or the measure of the set $M(z, N, A)$ in $x$ for which

$$
\left|\sum_{z}^{z+N} f\left(n_{k} x\right)\right|>A \cdot N
$$

is less than

$$
c / A^{2}(\log N)^{1+e / 2} \text {. }
$$

Consider the sets

$$
\begin{aligned}
& M\left(1,2^{n}, \delta\right) ; M\left(2^{n}, 2^{n-1}, 2 \delta / 2^{2}\right) ; \\
& M\left(2^{n}, 2^{n-2}, 4 \delta / 3^{2}\right), M\left(2^{n}+2^{n-1}, 2^{n-2}, 4 \delta / 3^{2}\right) ; \cdots
\end{aligned}
$$


There are $2^{k-1}$ sets of order $k$, that is, sets of the form

$$
M\left(2^{n}+2 u 2^{n-k}, 2^{n-k}, \delta 2^{k} /(k+1)^{2}\right), 0 \leqq u<2^{k-1} .
$$

From (21) it follows that the measure of any set of order $k$ does not exceed

$$
c(k+1)^{4} / \delta^{2} 2^{2 k}(n-k)^{1+\epsilon / 2} .
$$

Thus the measure of all the sets in (23) is less than $c(k+1)^{4} / \delta^{2} 2^{k}(n-k)^{1+\epsilon / 2}$, and the measure of all the sets $M_{n}$ in (22) does not exceed

$$
\sum_{k=0}^{n} \frac{c(k+1)^{4}}{\delta^{2} 2^{k}(n-k)^{1+\epsilon / 2}}<\frac{c_{1}}{\delta^{2} n^{1+\epsilon / 2}} .
$$

Thus

$$
\sum_{n=1}^{\infty} M_{n}<\infty
$$

But if $x$ does not belong to any of the sets (22) we have by a simple argument for all $2^{n} \leqq m<2^{n+1}$ (every $m$ is the sum of powers of 2 )

(25) $\left|\sum_{k=1}^{m} f\left(n_{k} x\right)\right|<\delta 2^{n}+\frac{\delta 2^{n}}{2^{2}}+\frac{\delta 2^{n}}{3^{2}}+\cdots+\frac{\delta 2^{n}}{k^{2}}+\cdots<2 \delta 2^{n} \leqq 2 \delta m$.

(24) and (25) clearly prove theorem $2\left(^{5}\right)$.

SYRACUSE UNIVERSITY, SyRACUSE, N. Y.

(5) The method used here is due to Hobson-Plancherel-Rademacher-Menchof. (See, for example, Rademacher, Math. Ann. vol. 87 (1922) p. 117-121.) 